\title{
Creating a serious game for people with visual impairment with an emphasis on adopting the smartphone
}

\author{
María del Rosario Peralta Calvo, Yusmar Alexis Flores Alvarado, Carlos \\ Manuel Santibáñez Camarillo
}

Published: 30 November 2020

\begin{abstract}
A great number of benefits that technology provides are accessed by a smartphone, in order to use it, it is necessary to know how to use the screen readers. However, the use of technology should not be limited by not seeing, not hearing or not being able to touch any technological device. There is currently a gap between people with visual impairment and technology. Despite the existence of a large number of projects that aim to reduce this gap in Mexico, it has not been consistently reduced. This motivates us to develop contributions in this area, so that people with visual disabilities enjoy the same benefits as sighted people. This paper aims to provide a literature review derived from the study phase and an outline of the design phase in the application of the User-Centered Design methodology. This methodology guides the development of a serious game project that will support visually impaired children in their first contact with a smartphone. The bibliographic review that is presented here has allowed us to contextualize and understand the impact of technological contributions and projects for the visually impaired.
\end{abstract}

\section{Keywords}

Visual Impairment, User-Centered Design, Serious Games, Accessibility.

\section{Introducción}

La presencia y necesidad de la tecnología es evidente, a tal grado de que tener un dispositivo tecnológico o acceso a un servicio se realice con apoyo de ésta. Dutta, Mía \& Geiger [8] mencionan que: "hoy vivimos en un mundo donde más personas tienen acceso a las Tecnologías de Información y Comunicación (TIC) (generalmente un teléfono móvil) que a los baños o agua limpia o red eléctrica". Sin embargo, los beneficios que la tecnología ofrece no están al alcance de todas las personas. Debido a la falta de desarrollo en accesibilidad, tal es el caso de las personas con discapacidad visual (PDV).

Peralta Calvo, M.R., Flores Alvarado, Y.A., Santibáñez Camarillo, C.M. Universidad de la Cañada

mperalta@unca.edu.mx,li_yusmar@unca.edu.mx,

carlosmsc@unca.edu.mx
El 3.6\% de la población con discapacidad visual en México se encuentra en Oaxaca, siendo el noveno estado con mayor presencia de personas con esta discapacidad, además de que hay una tendencia de aumento de las discapacidades a nivel mundial [17]. Por lo tanto, es crucial que todas las personas tengan acceso a la tecnología y puedan usarla, en caso contrario, habrá una marginación y exclusión social cada vez mayor [20]. Las PDV son probablemente las que se beneficien más de la tecnología.

Una gran parte de los beneficios que la tecnología otorga (acceso a internet, trámites bancarios, comercio, acceso a servicios de aplicaciones, redes sociales) son accedidos a través de teléfonos inteligentes, tabletas o computadoras. En el contexto de Oaxaca, el dispositivo más usado es el teléfono inteligente y el uso de internet es la actividad más frecuente [16].

En el contexto de Oaxaca, en la región Cañada, SAMM es el trabajo presenta una propuesta de acercar a las PDV con la tecnología. SAMM apoya al usuario en la tarea de reconocer el teclado. Mediante el prototipo de SAMM se buscó la forma de presentar al usuario una forma de aprendizaje entretenida, aplicando analogías a su edad para mejorar el aprendizaje. El niño estará divirtiéndose y también aprendiendo acerca del teclado. Esta tarea es fundamental puesto que para ocupar la computadora el usuario debe conocer el teclado [10]. El trabajo reportado en SAMM incluye una versión prototipo de un juego, que trajo resultados positivos para los NDV en las pruebas de usabilidad que reportaron.

Esto dio la pauta a que se fortaleciera la idea de optar por una opción entretenida y de aprendizaje para que las PDV y en específico los NDV puedan acercarse y usar la tecnología.

Un videojuego normal tiene muchos efectos positivos [14, 24, 26], pero un juego educativo (juego serio) aún más. Existen diversas definiciones de juego serio, pero la mayoría de ellas concuerda en que "están relacionados con el uso de juegos y tecnología de juegos para fines distintos al mero entretenimiento o diversión. Tales propósitos incluyen educación, capacitación, salud, etc." [31].

Por lo anterior, en las siguientes secciones se da a conocer la fase de estudio apoyándose en la revisión de literatura de proyectos e investigaciones que incluyeran la influencia de los videojuegos y juegos serios. Además de un primer bosquejo de la fase de diseño del proyecto del desarrollo de un juego serio para NDV en su primer contacto con el teléfono inteligente, aplicando la metodología diseño centrado en el usuario.

\section{Propuesta de solución}

Se pretende desarrollar un juego serio que apoye a los NDV en su primer contacto con un teléfono inteligente, que se diviertan, pero 
principalmente que aprendan a usar los gestos táctiles (más adelante mencionado solamente como gestos) básicos para usar un teléfono inteligente (acciones de deslizamientos y pulsaciones que se hacen en la pantalla del teléfono para acceder a opciones, menús y demás contenido del teléfono) [11]. Un juego serio cumple con ambos aspectos: aprendizaje y entretenimiento. Puede confundirse con un tutor inteligente, el cual adapta sus procesos de acuerdo al conocimiento del usuario y utiliza inteligencia artificial [12].

Las PDV usan teléfonos inteligentes por medio de los lectores de pantalla. El uso de lectores de pantalla ha crecido según el WebAim de un $12 \%$ en 2009 a un $88 \%$ en 2017 [33]. Al habilitar el lector de pantalla en un teléfono inteligente, la interacción cambia, pues para poder usar el teléfono es necesario utilizar gestos diferentes a los que comúnmente se usan (por ejemplo, al pulsar sobre un elemento, el lector lee el nombre del elemento y sus características, pero no accede al elemento. Para ello es necesario presionar dos veces consecutivamente). Cada lector de pantalla tiene gestos en particular, pero los gestos básicos son iguales. El juego serio pretende enfocarse a los gestos del lector de pantalla de los teléfonos con sistema operativo Android: Talkback. El juego serio pretende apoyar a los NDV a aprender tales gestos, estableciendo la interacción con el juego con base en estos.

\section{Metodología}

Esta propuesta se desarrolla bajo la metodología Diseño Centrado en el usuario (por sus siglas en inglés, UCD) [18]. Hasta el momento se tiene trabajado una parte de las primeras dos fases.

\subsection{Fase de Requerimientos}

En esta sección se presenta el estado del arte que guiará al estudio en esta primera etapa. Existen multitud de aportes que se han hecho para que PDV tengan acceso a la tecnología, pero las más usadas son: lector de pantalla JAWS, sitios web (que cumplen con estándares de accesibilidad) y teléfonos inteligentes (que vienen con opciones de accesibilidad) [15]. Esto es evidencia para desarrollar mejoras en relación a dispositivos móviles y la PDV. De manera preliminar se tiene contemplado como perfil de usuario a niños entre seis y doce años del estado de Oaxaca debido a que es importante que los NDV aprendan lo más pronto posible a usar la tecnología y Oaxaca es uno de los estados con mayor presencia de PDV [20].

\subsubsection{Estado del arte}

Se investigaron los proyectos enfocados a PDV mediante juegos serios para conocer el contexto de desarrollo y saber lo que existe en este ámbito. Existen distintos proyectos que pretenden acercar a las PDV a la tecnología, no se encuentra evidencia en estos de si las PDV presentan dificultad al usar los dispositivos o de cómo pueden ser usadas las aplicaciones por PDV que no han tenido contacto con la tecnología.

En un estudio donde evalúan veintiún juegos serios multimodales, la mayoría se enfoca en el apoyo a la orientación y movilidad, sólo dos aplicaciones (AudioGene y AudioNature) utilizan el modo de interacción de pantalla táctil y están enfocados a ciencias [7]. A su vez, los autores generaron una clasificación para el diseño y evaluación de juegos multimodales. En una investigación más se buscó desarrollar un juego serio enfocado a cálculos binarios siguiendo una lista de veinticinco requerimientos para desarrollar un juego serio accesible [29]. Leporini \& Palmucci [21] proponen un prototipo de juego serio enfocado a ejercicios / preguntas a través de la combinación de lector de pantalla e interacción basada en gestos donde el perfil de usuario comprende personas con y sin discapacidad visual. Sus resultados fueron satisfactorios, sin embargo, las PDV tuvieron problemas con el diseño de interfaz y el modo de interacción en algunos contenidos. Otros autores desarrollaron un juego serio enfocado a capacitación en orientación y movilidad convirtiendo actividades repetitivas en algo entretenido [27]. Se destaca el conocimiento previo que debe tener el usuario en el edificio en donde se llevará a cabo el juego. Morelli, Foley, Columna, Lieberman, \& Folmer [23] realizaron una versión modificada del juego VI Tennis en donde adaptaron señales vibrotáctiles y de audio como medios de interacción que permitiera a las PDV usar el juego con un enfoque de promover la actividad física.

Dentro de la investigación se encontraron artículos que proponen una serie de principios y recomendaciones en lo que respecta a juegos serios y PDV, de los cuales se consideran algunos para ser aplicados en este proyecto en función del objetivo del juego serio y el perfil del usuario.

Un estudio buscaba analizar si los juegos serios eran adecuados para PDV en cuanto a rehabilitación, y después de exponer el potencial de los juegos, propone una lista de recomendaciones [20]. Se buscará cumplir con una correcta definición de usuarios, objetivo adecuado al perfil de usuario, estructura desglosada de actividades para cumplir el objetivo, usar la tecnología como facilitador, cumplimiento de estándares, opciones de personalización y retroalimentación multimodal.

Una investigación revela la gran pasión que tienen las PDV por los videojuegos, y considera varios principios y recomendaciones a tener en cuenta al desarrollar un juego para estos usuarios [2]. Entre los que consideraremos: equilibrio entre proporcionar suficiente sonido para el juego y complicar demasiado el proceso requerido para comprender el estado del juego, formas nuevas e imaginativas de reducir las barreras al juego, representación de una PDV en la interfaz y sonidos únicos para los jugadores basados en elementos específicos del juego.

Otros autores exponen técnicas para desarrollar las historias de los juegos con el fin de asegurar la libertad en la interacción por el usuario [22]. Se piensa seguir los seis pasos iterativos para optimizar la interactividad que proponen los autores que son: encontrar base, marcar la intensidad narrativa, simplificar, diseñar escenas educativas, escribir historia sobre escenas de la estructura y evaluar historia.

En otra investigación se proponen herramientas de análisis para quienes desean desarrollar juegos serios móviles, donde destacan una nueva clasificación de pautas para analizar el nivel de accesibilidad de juegos (entre ellos, juegos serios) [19]. Se tiene como objetivo alcanzar al menos el nivel medio de accesibilidad donde pretendemos cumplir las siguientes pautas: modo de alto contraste, retroalimentación auditiva explícita, posibilidad de repetición, sin información fuera de la línea visual del jugador, guardar ajustes, desactivar la animación de fondo, apagar/encender elementos gráficos.

Greitzer, Kuchar \& Huston [13] recomiendan aplicar principios cognitivos, de aprendizaje y pedagógicos que aseguren el aprendizaje.

Por otro lado, en la investigación sobre PDV, se identificó una cantidad abrumadora de aplicaciones móviles que les ayudan a superar muchas barreras $[32,25]$. Entre las más populares en Android están: BeMyEyes, SuperSense, SuperVision, TapTapSee y Braille Tutor. En la Tabla 1 se muestra una comparación de estas aplicaciones con el juego serio que se propone en este artículo, al cual llamaremos "juego serio propuesto". Las comparaciones se centran en el objetivo principal de la aplicación. La retroalimentación consiste en la forma en que la aplicación reacciona, responde $\mathrm{u}$ opina al usuario conforme éste va 
interactuando con el teléfono. La independencia consiste en no necesitar a una persona más para poder llevar a cabo sus actividades. Es importante mencionar que para usar las aplicaciones de la PlayStore es necesario tener conocimiento previo en el uso de teléfonos inteligentes.

Tabla 1. Aplicaciones enfocadas a PDV.

\begin{tabular}{|c|c|c|c|}
\hline Aplicación & Objetivo & $\begin{array}{l}\text { Retroalim } \\
\text {-entación }\end{array}$ & Independencia \\
\hline BeMyEyes & $\begin{array}{l}\text { Apoyo cuando } \\
\text { no se tiene a } \\
\text { alguien al lado } \\
\text { en alguna duda }\end{array}$ & Inmediata & $\begin{array}{l}\text { Alta } \\
\text { independencia }\end{array}$ \\
\hline SuperSense & $\begin{array}{l}\text { Apoyo en } \\
\text { detecciones de } \\
\text { objetivos } \\
\text { generales o } \\
\text { específicos, y } \\
\text { lectura de texto. }\end{array}$ & $\begin{array}{l}\text { Inmediata. } \\
\text { Mensajes } \\
\text { largos. }\end{array}$ & $\begin{array}{l}\text { Alta } \\
\text { independencia }\end{array}$ \\
\hline SuperVision & $\begin{array}{l}\text { Apoyo para } \\
\text { PDV moderada } \\
\text { para ver objetos } \\
\text { o textos usando } \\
\text { zoom, cambio } \\
\text { de contrastes, } \\
\text { etc. }\end{array}$ & Inmediata. & Moderada \\
\hline TapTapSee & $\begin{array}{l}\text { Detección de } \\
\text { objetos. Y } \\
\text { lectura de sus } \\
\text { detalles. }\end{array}$ & $\begin{array}{l}\text { Inmediata } \\
\text { y clara. }\end{array}$ & Alta \\
\hline $\begin{array}{l}\text { Juego serio } \\
\text { propuesto }\end{array}$ & $\begin{array}{l}\text { Apoyo en el } \\
\text { aprendizaje del } \\
\text { uso del teléfono } \\
\text { inteligente }\end{array}$ & $\begin{array}{l}\text { Inmediata } \\
\text { y clara }\end{array}$ & $\begin{array}{l}\text { Baja al inicio } \\
\text { inmediato. } \\
\text { Después habrá } \\
\text { una alta } \\
\text { independencia }\end{array}$ \\
\hline
\end{tabular}

Hemos visto hasta el momento, aplicaciones semejantes en muchos puntos excepto en el objetivo de ser un apoyo a los NDV en su primer contacto con teléfonos inteligentes. En la investigación se identificaron hasta el momento dos proyectos que llevan el mismo objetivo, los cuales se presentan a continuación.

El primer proyecto consiste en un tutor físico, es decir, una organización en donde capaciten a los NDV a usar los dispositivos tecnológicos. El Centro de Estudios para Invidentes [6] dentro de sus planes tiene tecnología asistiva en varios dispositivos, entre ellos, los teléfonos inteligentes. Cabrera, García \& Suárez [5] publicaron un manual para personas que asesoran a PDV en el uso de tecnología. En tal manual plantean 10 pasos para lograr este objetivo en el dispositivo de computadora. La experiencia de los NDV en su primer contacto y la retroalimentación dada depende del modo en que el profesional apoye en el aprendizaje. Inicialmente, los NDV no tienen la opción de aprender de manera independiente y segura. Una ventaja de este proyecto es que los profesionales ayudan a superar las barreras que se vayan encontrando los NDV en el transcurso de su aprendizaje, aunque tal aprendizaje depende de estar físicamente con el profesional. Los NDV no necesitan tener conocimiento previo alguno para empezar a aprender.

El segundo proyecto, es el lector de pantalla Talkback, que tiene dentro de sus opciones un tutorial de cómo usar el lector a fin de que el usuario pueda usar el teléfono inteligente. Sin embargo, Rodríguez, Montague, Nicolau \& Guerreiro [28] analizaron la experiencia de las PDV en su primer contacto con un teléfono inteligente, y dentro de ello se comprendía el tutorial de Talkback. Llegaron a la conclusión de que este sistema no era suficiente para preparar a las PDV en su uso del teléfono inteligente, presentaron varios problemas con el tutorial. Los usuarios expresaron que sin alguna persona de apoyo aumenta el grado de dificultad para el uso de este tipo de dispositivos. Hay una falta de retroalimentación. Este proyecto es prometedor en lograr una mayor independencia de los NDV pero con los detalles mencionados tal objetivo es difícil de cumplir. Según los autores, las barreras de los usuarios solo cambiaron de forma. A pesar de esta situación, los usuarios estaban dispuestos a seguir explorando y mejorar su habilidad para usar estos dispositivos debido a los beneficios que tendrían al utilizarlos. En base a estos autores se puede decir que el tutorial no es suficiente para preparar a los NDV en su primer contacto.

Por su parte, el juego serio propuesto será desarrollado para lograr un primer contacto estableciendo confianza hasta un nivel de deseo por el juego, retroalimentación inmediata con lenguaje sencillo, claro y alentador. Pretende brindar independencia al usuario para que pueda practicar en cualquier momento sin un conocimiento previo en el uso de teléfonos inteligentes.

Cada proyecto revisado tiene diferentes contextos, perfiles de usuario, objetivos y tareas. Además, todos ellos son fascinantes y han aportado de manera importante en beneficio a las PDV. Stephanidis [30] recomienda para el desarrollo de software para PDV un enfoque en la interacción basada en habla, hápticos, seguimiento de gestos y cabeza. En el juego serio buscamos lograr nuestro objetivo enfocándonos en las habilidades de los usuarios.

El juego serio propuesto tiene la ventaja de no asumir que los NDV saben utilizar un teléfono inteligente y por lo tanto, poder brindar un primer contacto satisfactorio. También promueve la independencia y pretende brindar una retroalimentación corta y concreta. La experiencia de usuario es una parte clave en el desarrollo de este juego serio, cumpliría los parámetros de aprendizaje y entretenimiento, con esto se pretende que el usuario opte por usar un dispositivo tecnológico y que esté a su alcance en cualquier momento.

Continuando con la siguiente fase de la metodología UCD se anticipan avances preliminares en el diseño.

\subsection{Fase de diseño}

En seguida se presentan algunas generalidades de la fase de diseño.

\subsubsection{Avances preliminares}

Tomando los símbolos de Edwards [9] se diseñó un diagrama que ilustra lo que el juego serio propuesto pretende hacer (ver Figura 1). El sistema es el teléfono inteligente, la adaptación es el lector de pantalla y el usuario son las PDV. El punto aquí es lograr el acoplamiento de la adaptación con el usuario. Esto ocurre cuando el sistema no es accesible. Pero tomando la premisa de que muchas personas alrededor del mundo saben usar los teléfonos inteligentes, nuestro objetivo es asegurar tal acoplamiento por medio del juego serio (Fig. 1.b). El juego serio será un apoyo para lograr la independencia, buena retroalimentación y un mejor manejo de las muchas incertidumbres al usar por primera vez un teléfono inteligente. Se anticipará una parte importante de la experiencia que tendrán los NDV cuando estén fuera del juego evitando así muchas frustraciones y dando las herramientas para que puedan practicar de modo que no tengan problemas. 
a)

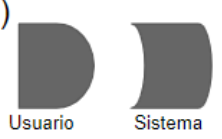

b)

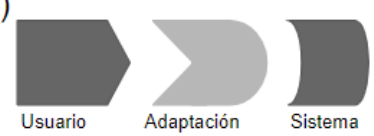

c)

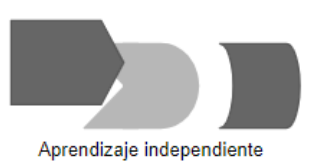

d)

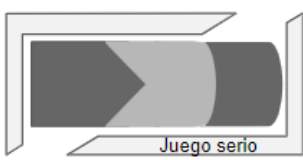

Aprendizaje con apoyo de juego serio propuesto

Figura 1. a) El sistema encaja con las habilidades de los usuarios. b) El sistema no encaja con las habilidades del usuario como se supuso, entonces es necesaria una adaptación para poder ser usado. c) En el proceso de aprendizaje de la adaptación, no se logra el objetivo. d) El juego serio pretende asegurar el cumplimiento del objetivo de la adaptación.

La base del juego estará centrada en los gestos mostrados en la Tabla 2 [11]. Se planea que el avance en el juego sea progresivo en dificultad y funciones, es decir, de niveles sencillos a niveles difíciles, de gestos básicos a gestos con grados de dificultad.

Tabla 2. Gestos de Talkback de Android. [11]

\begin{tabular}{|l|l|l|}
\hline No. & Gesto & Acción en teléfono \\
\hline 1 & Un toque & Selección de elemento \\
\hline 2 & Deslizar hacia la derecha & Elemento siguiente \\
\hline 3 & Deslizar hacia la izquierda & Elemento anterior \\
\hline 4 & $\begin{array}{l}\text { Deslizar hacia arriba o } \\
\text { hacia abajo }\end{array}$ & $\begin{array}{l}\text { Desplazarse por la } \\
\text { configuración de } \\
\text { navegación }\end{array}$ \\
\hline 5 & Doble toque & $\begin{array}{l}\text { Seleccionar elemento } \\
\text { enfocado }\end{array}$ \\
\hline 6 & Arriba luego abajo & $\begin{array}{l}\text { Mover al primer } \\
\text { elemento de la pantalla }\end{array}$ \\
\hline 7 & $\begin{array}{l}\text { Mover al último elemento } \\
\text { de la pantalla }\end{array}$ & \begin{tabular}{l} 
Abajo y luego arriba \\
\hline
\end{tabular}
\end{tabular}

La manera en que el juego serio garantiza ser un apoyo en el aprendizaje, es por medio del aprendizaje por descubrimiento como se intentó en investigaciones pasadas en computadoras, planteando los conceptos de las funciones de las teclas por medio de acciones en un juego semejante $[1,10,4]$. Por ejemplo, para dar a entender el concepto de que la tecla Enter sirve para acceder a carpetas/archivos/links/etc, en el juego el usuario debería pulsar la tecla Enter para entrar a una habitación, confirmar compras, entre otras acciones, dando énfasis al concepto. Esto mismo se pretende hacer con el juego serio para teléfonos inteligentes, diseñando el juego de tal manera que la dinámica de éste sea acorde a los gestos estándar del lector de pantalla.

\section{Conclusión}

Dada la complejidad del teléfono inteligente en PDV, surge la necesidad de asegurar el acoplamiento de la adaptación con las habilidades de los usuarios. Por su parte, el juego serio enfocará su atención en asegurar el primer contacto siguiendo los principios y recomendaciones mencionados en este artículo. Con esto, el usuario podrá usar un teléfono inteligente y por lo tanto, tener acceso a todos los proyectos, aplicaciones y propuestas desarrolladas para las PDV. Como se mencionó, esta investigación estará guiada por el estudio del arte, por lo que con este se procederá a llevar a cabo las siguientes etapas hasta a completar una iteración de la metodología UCD.

\section{Referencias}

[1] Adelia, W. S., and Surya, E. Resolution to Increase Capacity by Using Math Students Learning Guided Discovery Learning (gdl). Inter. J. of Sciences: Basic and Applied Research. (2017), 144-154.

[2] Andrade, R., Rogerson, M. J., Waycott, J., Baker, S., and Vetere, F. Playing Blind: Revealing the World of Gamers with Visual Impairment. Proc. CHI 19, ACM Press (2019), $1-14$.

[3] Archambault, D., Ossmann, R., Gaudy, T. and Miesenberger, K. Computer Games and Visually Impaired People. Upgrade, 8, 2 (2007).

[4] Baro Cálciz, A. Metodologías activas y aprendizaje por descubrimiento. Innovación y experiencias educativas. 2011.

[5] Cabrera Martínez, J., García Rodríguez, A., and Suárez García, J. I. (2015). Gobierno de México.

https://www.gob.mx/cms/uploads/attachment/file/127691/ma nual_para_asesor_pcdv.pdf

[6] Centro de Estudios para Invidentes. Tecnología asistiva. http://www.ceiacmexico.org/tecnologiaasist.html

[7] Darin, T., Sánchez, J., and Andrade, R. Dimensions for the design and evaluation of multimodal video games for the cognition of people who are blind. Proc. IHC '15, ACM Press (2015), 1-4.

[8] Dutta, S., Mia, I. and Geiger, T. The Networked Readiness Index 2010-2011: Celebrating 10 Years of Assessing Networked Readiness. 2011 World Economic Forum.

[9] Edwards, A. D. N. Computers and people with disabilities. In Extraordinary Human-Computer Interaction: Interfaces for Users with Disabilities, A. D. N. Edwards Ed., Cambridge University Press (1995), 19-43.

[10] Flores Alvarado, Y. A., Jiménez Mía, N., Arriaga Fierro, A., Peralta Calvo, M. and Hernández Paxtián, Z. J. Hacia un sistema parlante para personas ciegas y de baja visión. Avances en Interacción Humano-Computadora (2018).

[11] Google Help. Android Accesibility Help. https://support.google.com/accessibility/android/answer/6151 827

[12] Gordillo-Guillén, A., Andrade, H., and Rivera-Lopez, R. Modelo de un sistema tutor inteligente para el desarrollo del pensamiento computacional. Journal CIM . (2017) 
[13] Greitzer, F. L., Kuchar, O. A., and Huston, K. Cognitive science implications for enhancing training effectiveness in a serious gaming context. J. Educ. Resour. Comput. 7, 3 (2007), 2-es.

[14] Gros, B. Digital Games in Education: The Design of GameBased Learning Environments. J Res Tech Educ. 40. (2006).

[15] Inclúyeme. Todo lo que necesitas saber sobre Discapacidad Visual https://www.incluyeme.com/todo-lo-que-necesitassaber-sobre-discapacidad-visual/

[16] Instituto Federal de Telecomunicaciones. Uso de las TIC y las actividades por internet en México: Impacto de las características sociodemográficas de la población. 2018. $\mathrm{http}$ //www.ift.org.mx/sites/default/files/contenidogeneral/est adisticas/usodeinternetenmexico.pdf

[17] Instituto Nacional de Estadística y Geografía. La discapacidad en México, datos al 2014. 2014. http://internet.contenidos.inegi.org.mx/contenidos/Productos/ prod_serv/contenidos/espanol/bvinegi/productos/nueva_estru c/702825090203.pdf

[18] Interaction Design Foundation. User Centered Design. https://www.interaction-design.org/literature/topics/usercentered-design

[19] Jaramillo-Alcázar, A., and Luján-Mora, S. Mobile serious games: An accessibility assessment for people with visual impairments. Proc. Teem 2017, ACM Press (2017), 1-6.

[20] Leporini, B. and Hersh, M. Games for the rehabilitation of disabled people. Proc. REHAB 16, ACM Press (2016), 109112.

[21] Leporini, B. and Palmucci, E. A Mobile Educational Game Accessible to All, Including Screen Reading Users on a Touch-Screen Device. Proc. mLearn 2017, ACM Press (2017), 1-4.

[22] López-Arcos, J. R., Gutiérrez Vela, F. L., Padilla-Zea, N., and Rodríguez, P. P. Interactive narrative design for geolocated experiences. Proc. Interacción 16, ACM Press (2016), 1-2.
[23] Morelli, T., Foley, J., Columna, L., Lieberman, L., and Folmer, E. VI-Tennis: A vibrotactile/audio exergame for players who are visually impaired. Proc. FDG 10, ACM Press (2010), 147-154.

[24] Oei, A. C. and Patterson, M. D. (2013). Enhancing Cognition with Video Games: A Multiple Game Training Study. PLoS ONE 8, 3 (2013).

[25] Pérez, E. Accesibilidad en Android: 21 aplicaciones para personas ciegas o con problemas de visión. 2018. xatakandroid.com/aplicaciones-android/accesibilidad-enandroid-21-aplicaciones-para-personas-invidentes

[26] Prensky, M. The Seven Games of Highly Effective People. Microsoft Games for Windows. 2004.

[27] Regal, G., Mattheiss, E., Sellitsch, D., and Tscheligi, M. Mobile location-based games to support orientation \& mobility training for visually impaired students. Proc. MobileHCI 18, ACM Press (2018), 1-12.

[28] Rodrigues, A., Montague, K., Nicolau, H., and Guerreiro, T. Getting Smartphones to Talkback: Understanding the Smartphone Adoption Process of Blind Users. Proc. ASSETS 15, ACM Press (2015), 23-32.

[29] Salvador Ullauri, L., Jaramillo Alcázar, A., and Luján Mora, S. A Serious Game Accessible to People with Visual Impairments. Proc. ICETC 2017, ACM Press (2017). 84-88.

[30] Stephanidis, C. Design for All. https://www.interactiondesign.org/literature/book/the-encyclopedia-of-humancomputer-interaction-2nd-ed/design-4-all

[31] Susi, T., Johannesson, M., and Backlund, P. Serious Games An Overview. Technical Report HS- IKI -TR-07-001. 2007

[32] Tuke, H. Useful Resources For Blind and Visually Impaired People. 2016. https://lifeofablindgirl.com/2016/04/11/usefulresources-for-blind-and-visually-impaired-people/

[33] WebAim. Screen Reader User Survey \#7 Results. 2017. https://webaim.org/projects/screenreadersurvey7/

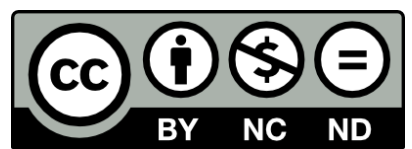

(C) 2020 by the authors. This work is licensed under the Creative Commons AttributionNonCommercial-NoDerivatives 4.0 International License. To view a copy of this license, visit http://creativecommons.org/licenses/by-nc-nd/4.0/ or send a letter to Creative Commons, PO Box 1866, Mountain View, CA 94042, USA. 